\title{
Thoraco-esophageal fistula during percutaneous tracheostomy
}

\author{
Yusuke Asakura ${ }^{1} \mathbb{C}$
}

Received: 26 May 2018 / Accepted: 28 June 2018 / Published online: 3 July 2018

(c) Japanese Society of Anesthesiologists 2018

Keywords Thoraco-esophageal fistula $\cdot$ Percutaneous tracheostomy

To the Editor:

Percutaneous tracheostomy (PT) has now been performed in a majority of patients as an alternative to the conventional surgical tracheostomy (ST) [1]. The advantages of PT over ST include its fast, simple and the minimal invasive procedures, which subsequently decrease the frequency of surgical site infections (SSIs) relating to the procedure. However, although infrequent, serious complications have occasionally been met with minor bleeding with its highest frequency followed by rare but life-threatening complications, such as thoraco-innominate artery fistula and the thoraco-esophageal fistula (TEF) formation. These serious complications are usually caused by the damage to the posterior tracheal wall during the insertion of the puncture needles with an inappropriate depth of the puncture. In an attempt to better understand the anatomical structures of the tissues surrounding the trachea, the cervical MRI images from 60 Japanese adult males and 60 females were randomly selected after an
IRB approval was obtained. The depth from the skin to the posterior tracheal wall at the first-second cricoid ring level was measured. The average age of 60 males and 60 females was 65.8 years (range $37-91$; median 67.5 ) and 74.6 years (range 43-92; median 77). The average depth from the skin to the posterior wall of the trachea in males and females was $26.35 \mathrm{~mm}$ (range 18.26-44.4; median 25.88) and $23.47 \mathrm{~mm}$ (range 14.17-33.88; median 22.23), respectively. Based on the results, we have marked the puncture needle at $15 \mathrm{~mm}$ from the point before the insertion of the needles, and we have been successfully performing PT without any complications by avoiding the inappropriate depth of the puncture.

\section{Reference}

1. Mehta C, Mehta Y. Percutaneous tracheostomy. Ann Card Anaesth. 2017;20:19-25.
Yusuke Asakura

yasakura@kaikou.or.jp

1 Department of Anesthesiology, Nagoya Kyoritsu Hospital, 1-172, Hokke, Nakagawa-ku, Nagoya, Aichi, Japan 